\title{
Discrete Optimization on Unsteady Pressure Fluctuation of a Centrifugal Pump Using ANN and Modified GA
}

\author{
Wenjie Wang \\ Jiangsu University \\ Qifan Deng \\ Jiangsu University \\ Ji Pei ( $\nabla_{\text {jpei@ujs.edu.cn ) }}$ \\ Jiangsu University \\ Jinwei Chen \\ Jiangsu University \\ Xingcheng Gan \\ Jiangsu University
}

Original Article

Keywords: Centrifugal Pump, Unsteady Performance Optimization, Discrete Design Variable, Discrete Genetic Algorithm

Posted Date: November 15th, 2021

DOI: https://doi.org/10.21203/rs.3.rs-1059081/v1

License: (c) (1) This work is licensed under a Creative Commons Attribution 4.0 International License. Read Full License 


\section{Title page}

\section{Discrete optimization on unsteady pressure fluctuation of a centrifugal pump using ANN and modified GA}

Wen-Jie Wang, born in 1990, is currently an associate researcher at Jiangsu University, China. He received his PhD degree from Jiangsu University, China, in 2018. His research interests include intelligent optimization algorithm improvement, hydraulic optimization design of fluid machinery, measurement and analysis of unsteady flow in pumps, and transient flow characteristics of pump turbine.

E-mail: wenjiewang@ujs.edu.cn

Qi-Fan Deng, born in 1994, is currently a $\mathrm{PhD}$ candidate at National Research Center of Pumps, Jiangsu University, China. He received his master degree from Jiangsu University, China, in 2020. His research interests include pump optimization and unstable flow in pump

E-mail: deng_qf@hotmail.com

Ji Pei, born in 1984, is currently a researcher at Jiangsu University, China. He received his PhD degree from Jiangsu University, China, in 2013. His research interests include fluid machinery (pump) advanced optimization design method, internal flow theory, and multidisciplinary coupling analysis.

Tel: +86-511-88783358; E-mail: jpei@ujs.edu.cn

Jin-Wei Chen, born in 1995, is currently a master candidate at National Research Center of Pumps, Jiangsu University, China.

E-mail: cjwszz@foxmail.com

Xing-Cheng Gan, born in 1994, is currently a $\mathrm{PhD}$ candidate at National Research Center of Pumps, Jiangsu University, China. He received his master degree from Jiangsu University, China, in 2019. His research interests include pump system optimization.

E-mail: ganxingcheng@gmail.com

\section{Corresponding author: Ji Pei E-mail: jpei@ujs.edu.cn}




\title{
Discrete optimization on unsteady pressure fluctuation of a centrifugal pump using ANN and modified GA
}

\author{
Wen-Jie Wang • Qi-Fan Deng • Ji Pei • Jin-Wei Chen • Xing-Cheng Gan
}

Received June xx, 201x; revised February xx, 201x; accepted March xx, 201x

(C) Chinese Mechanical Engineering Society and Springer-Verlag Berlin Heidelberg 2017

\begin{abstract}
'Abstract: Pressure fluctuation due to the rotor-stator interaction in turbomachinery is unavoidable, inducing strong vibration and even shortening the lifecycle. The investigation on optimization method of an industrial centrifugal pump was carried out to reduce the pressure fluctuation intensity. Considering the time-consuming transient calculation of unsteady pressure, a novel optimization strategy was proposed by discretizing design variables and genetic algorithm. Four highly related design parameters were chosen, and 40 transient sample cases were generated and simulated using an automatic simulation program. Furthermore, a modified discrete genetic algorithm (MDGA) was proposed to reduce the optimization cost by unsteady simulation. For the benchmark test, the proposed MDGA showed a great advantage over the original genetic algorithm in terms of searching speed and could deal with the discrete variables effectively. After optimization, an improvement in terms of the performance and stability of the inline pump was achieved.

Keywords: Centrifugal Pump - Unsteady Performance Optimization - Discrete Design Variable - Discrete Genetic Algorithm
\end{abstract}

\section{Introduction}

Centrifugal pumps as a kind of fluid machinery, are widely used in irrigation, petroleum, chemical, and nuclear power fields. Wherever there is liquid flow, the pumps work. The vertical inline pump is a special kind of centrifugal pump with an elbow-shaped inlet structure, which is widely utilized as a solution to limited installation space such as urban water supply and ship transportation[1]. However, the curved inlet channel deteriorates the flow conditions in

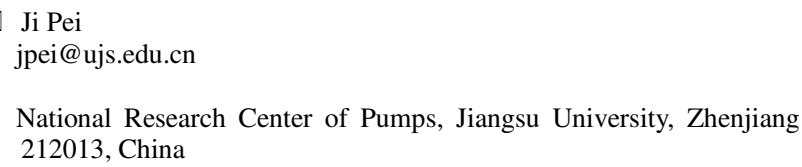

the inline pump, which results in the decrease of the hydraulic performance and the increase of damage risk. Pressure fluctuation is one significant factor that affects operating stability and efficiency. It will increase vibration but cannot be eliminated even under design condition[2,3]. Therefore, the optimizing design of the vertical inline pump is of great significance for energy-saving as well as safe operation issues.

The efficiency optimization is mainly based on steady simulation to save time, although transient simulation is more accurate. However, transient simulation is necessary when simulate pressure fluctuation and enough simulation time is also significant to improve the resolution of time and frequency, which is time consuming. Hence, appropriate optimization methods should be selected for the reduction of pressure fluctuation.

In recent years, many researchers tried to reduce pressure fluctuation in centrifugal pumps. Fu et al. [4] investigated the influence of stagger angle of a double-entry impeller on pressure fluctuation and found the optimized arrangement of the impeller. Li et al. [5] modified the leading edge of a pump-turbine impeller (working at pump mode) to reduce the pressure fluctuation in hump region. Because of limitation of variables and samples, the optimal solution might not be achieved. The optimization with multiple parameters is necessary to reduce pressure fluctuation inside centrifugal pumps.

Nowadays, benefitting from the development of computational resources, optimization methods combining parametric design, CFD prediction, surrogate models, and intelligent algorithms are broadly applied to improve the performance of pumps. At the same time, many studies have confirmed the feasibility and stability of this kind of approach[6-10].

The common surrogate models used in the optimizing 
design of pumps include response surface model (RSM)[6,9,11], artificial neural network (ANN) [7,12-14], Kriging model $[8,15]$, etc. To compare the capacity of different models, Pei et al. [16] presented an optimization work for an industrial centrifugal pump by using ANNs with different structures. The results indicated that the relationship between the decision variables (e.g., impeller design variables) and the objective functions (e.g., efficiency) shows strong nonlinearity; thus, the ANNs with multiple hidden layers usually perform better than simple feedforward networks. A similar conclusion is also obtained by Demeulenaere et al. [17] that the ANN with two hidden layers had better accuracy in predicting multi-peak functions.

On the other hand, the pump optimization problems usually show great complexity and cost much on the computational resource, especially those who using CFD technique as the objective functions[18]. Therefore, improving the convergence speed of the algorithms is essential to reduce computational costs and improve optimization efficiency. Many studies have been reported to improve the algorithm performance to solve complicated optimization problems (e.g., improving cavitation performance) by modifying the existed algorithms (e.g., Genetic Algorithm [19,20], Particle Swarm Optimization[21]). Nourbakhsh et al. [22] presented a research which compared the performance of NSGA-II and MOPSO for a centrifugal pump optimization problem, which indicated that the PSO algorithm produced better results than GA.

However, although the algorithms have been significantly improved by the efforts of many scholars, the performance for solving the optimization problems with large computational volumes is still unsatisfactory. Thus, the current pump optimization schemes mainly focus on improving the efficiency, head, and other steady characteristics [23-26] The unsteady parameters such as pressure fluctuation intensity are not considered because of the enormous computations required through the optimization procedures. For this situation, besides increasing computational resources, the best way is to reduce the sample quantity requirement. To reduce the unsteady optimization period, two aspects are considered: the design variables are discrete, and the novel discrete genetic algorithm is proposed to solve the discrete optimization problems.

In this research, the pressure fluctuation intensity near the tongue of an industrial vertical inline pump was considered in the optimization process. Four design parameters of the impeller blades were selected.
Meanwhile, a modified discrete genetic algorithm (MDGA) was proposed to reduce the sample requirement. Finally, a comparison of performance and flow conditions between the original case and the optimized case was carried out to further study the reasons for performance improvement.

\section{Methodology}

\subsection{Numerical methodology}

\subsubsection{Computational model}

In this research, the impeller of an industrial inline pump (specific speed $n_{\mathrm{s}}=132$, in Chinese standard, the definition is given as Eq. (1)) was optimized, and the characteristics of the original case, sample cases, and the optimized case were predicted by numerical simulations. As shown in Fig. 1 , the flow domain was divided into five parts in the CFD calculations, includes suction pipe, curved inlet pipe, impeller, volute, and discharge pipe. The design flowrate $Q_{\mathrm{d}}$ is $50 \mathrm{~m}^{3} / \mathrm{h}$, the design head $H_{\mathrm{d}}$ is $20 \mathrm{~m}$, and the nominal rotation speed $\mathrm{n}$ is $2910 \mathrm{rpm}$. Other geometry parameters of the original model are listed in Table. 1.

$$
n_{s}=\frac{3.65 n \sqrt{Q_{\mathrm{d}}}}{H_{\mathrm{d}}^{3 / 4}}
$$

Where $n_{\mathrm{s}}$ is specific speed, $n$ is nominal rotation speed, $Q_{\mathrm{d}}$ is design flowrate, and $H_{\mathrm{d}}$ is design head.

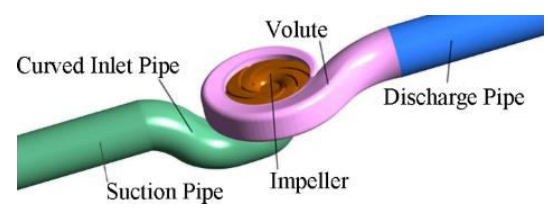

Figure 1 Computational domain

Table 1 Original geometry parameters

\begin{tabular}{cccc}
\hline Parameter Name & Abbreviation & Value & Unit \\
\hline Pump inlet diameter & $D_{\mathrm{s}}$ & 80 & $\mathrm{~mm}$ \\
Pump outlet diameter & $D_{\mathrm{d}}$ & 80 & $\mathrm{~mm}$ \\
Impeller inlet diameter & $D_{1}$ & 72 & $\mathrm{~mm}$ \\
Impeller outlet diameter & $D_{2}$ & 136 & $\mathrm{~mm}$ \\
Impeller inlet width & $b_{1}$ & 34.5 & $\mathrm{~mm}$ \\
Impeller outlet width & $b_{2}$ & 17.8 & $\mathrm{~mm}$ \\
Impeller blade number & $z$ & 6 & \\
Impeller blade angle at the & $\beta_{1}$ & 38 & degree \\
leading edge & & & \\
Impeller blade angle at the & $\beta_{2}$ & 23 & degree \\
\hline trailing edge & & &
\end{tabular}

\subsubsection{Grid distribution}

The structural grids of each domain shown in Fig. 1 were 
generated using the commercial pretreat software ANSYS ICEM CFD coupled with the multi-block strategy to improve the overall grid quality. The near-wall regions were refined to capture accurate near-wall flow behaviors, and the node number of each flow passage was determined by the grid sensitivity analysis.

The test results are listed in Table. 2, it could be observed that the computational head was going to be stable when the grid number increased by 4.29 million, so grid scheme $\mathrm{G}$ was chosen for further research, and the grid specifications are given in Table. 3. Meanwhile, the maximum $y+$ for the whole flow domain is less than 30 , and the figure for crucial areas such as leading-edge, tongue was less than 10 , which could meet the requirement of flow feature predictions in the pump by using shear stress transport k- $\omega$ turbulence model (SST).[28] The final grid conditions are shown in Fig. 2.

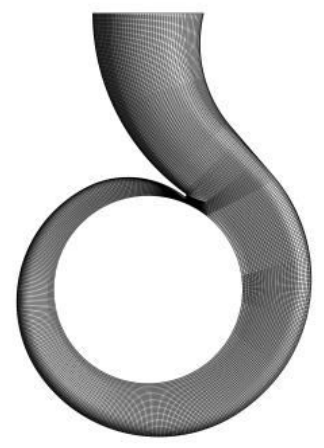

(a) Volute

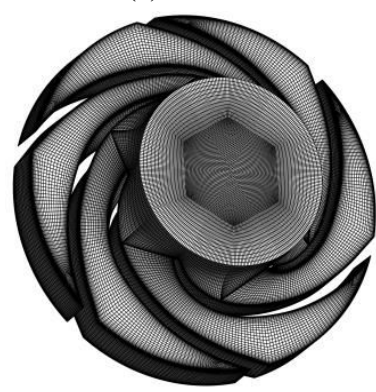

(b) Impeller

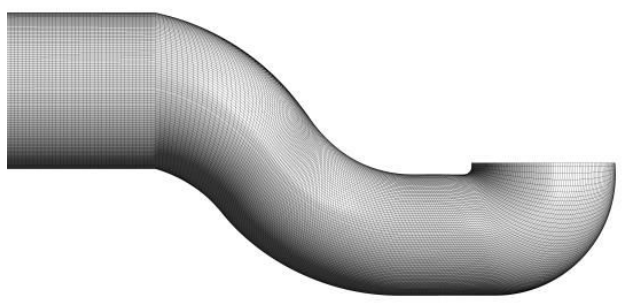

(c) Curved inlet pipe

Figure 2 Grid condition

Table 2 Grid sensitivity analysis

\begin{tabular}{ccc}
\hline Scheme index & Node number /million & Head $/ \mathrm{m}$ \\
\hline A & 0.976 & 20.122 \\
B & 1.126 & 19.698 \\
C & 1.434 & 19.776 \\
D & 1.817 & 20.185 \\
E & 2.387 & 20.196 \\
F & 3.482 & 20.245 \\
G & 4.290 & 20.275 \\
H & 6.603 & 20.281 \\
I & 8.390 & 20.284 \\
\hline
\end{tabular}

Table 3 Grid specifications

\begin{tabular}{cccccc}
\hline Component & $\begin{array}{c}\text { Suction } \\
\text { Pipe }\end{array}$ & $\begin{array}{c}\text { Inlet } \\
\text { Pipe }\end{array}$ & Impeller & Volute & $\begin{array}{c}\text { Discharge } \\
\text { Pipe }\end{array}$ \\
\hline $\begin{array}{c}\text { Nodes } \\
\text { number }\end{array}$ & 779,544 & $\begin{array}{c}581, \\
578\end{array}$ & 933,510 & $\begin{array}{c}1,216, \\
305\end{array}$ & 779,544 \\
\hline
\end{tabular}

\subsubsection{Numerical setup}

In this research, the three-dimensional Unsteady Reynolds-averaged Navier-Stokes equations (URANS) were solved by the commercial CFD code ANSYS CFX coupled with the SST k- $\omega$ turbulence model for the flow predictions of the original case, sample cases, and the optimized case[28]. The boundary conditions were set in accordance with the actual working conditions.

Specifically, the total pressure inlet with the value of 1 atm and the turbulence intensity of 5\% was set as the pump inlet condition, and the mass flowrate outlet was used to describe the pump outlet. All physical surfaces in the flow domains were set as no-slip walls with the roughness of $25 \mu \mathrm{m}[29]$. During the CFD calculation process, the solution for a steady state with the frozen stator strategy was conducted first and then utilized as the initial situation of the transient computation. For the transient cases, the interfaces between the rotating part and stationary domains were changed to the transient rotor-stator strategy, so the relative position between the impeller and the volute was updated at each timestep[30]. The timestep in the transient cases was set as $1.718 \times 10^{-4} \mathrm{~s}$, which is the time required for the impeller to rotate by 3 degrees. The typical time duration is set as $0.5155 \mathrm{~s}$, corresponding to the time consumption of 25 rotations. The average mathematical value of each characteristic parameter of the last rotation was used to describe the performance of the inline pump, and the pressure data of the last 15 rotations $(0.2062 \mathrm{~s}$ $0.5155 \mathrm{~s}$ ) was selected for the pressure fluctuation analysis.

Furthermore, in order to evaluate the pressure fluctuation intensity, a dimensionless pressure fluctuation intensity coefficient (PFIC) was defined as the Eq. (2). 
A monitor point V1 was added to obtain the pressure fluctuation features near the tongue (as shown in Fig. 3).

$$
C_{p}^{*}=\frac{\sqrt{\frac{1}{N} \sum_{j=0}^{N-1} \tilde{p}\left(\text { node }, t_{0}+j \Delta t\right)^{2}}}{\rho u_{2}^{2} / 2}
$$

where $N$ is the number of pressure samples in one rotation period, $\tilde{p}$ is the periodic pressure component, node is the grid node index, $t_{0}$ is the start of the rotation period, $\rho$ is the fluid density, and $u_{2}$ is the circumferential velocity at the impeller outlet.

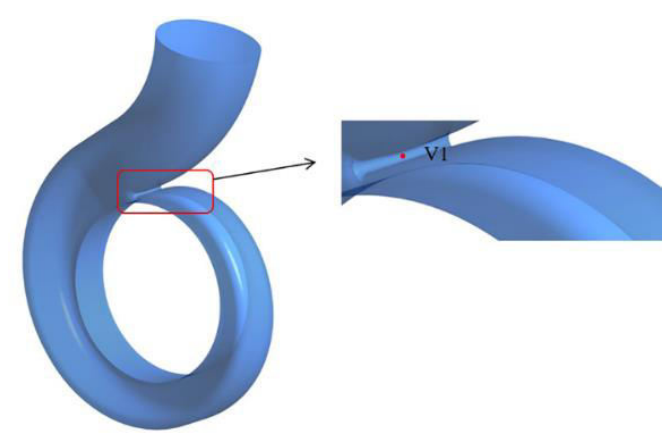

Figure 3 Location of monitor point at volute tongue

\subsection{Optimization procedure}

The overall procedure of the optimization for the inline pump is shown in Fig. 4, which could be mainly classified into four parts: optimization problem definition (Step 1 and Step 2), sample data set generation (Step 3 and Step 4), surrogate model training (Step 5 and Step 6) and solving (Step 7).

As mentioned in section 1.2, the optimization process based on transient sample cases costs much computational resources and time. Therefore, in this study, two approaches were applied to reduce the requirement of sample size. Firstly, it is well known that the accuracy of surrogate models is highly related to the samples, and the required sample size is related to the number of variables[18]. Hence, in Step 2, a data mining process was carried out to select highly correlated variables, and finally, four decision variables were chosen for the optimization process. On the other hand, the pump optimization problem does not require high convergence precision because the model precision is limited, and more iterations mean more computation costs. Thus, to fit this situation, a modified discrete genetic algorithm was proposed in Step 7 for fast convergence performance.

\subsubsection{Objective function}

In order to improve both the efficiency and the operating reliability of the inline pump, to minimize the pressure fluctuation coefficient at the nominal operating condition was selected as objective function. On the other hand, the efficiency and the head were utilized as the constraints through the optimization process to prevent the performance drop. The mathematical description of the objective function is given in the Eq. (3).

$$
\begin{aligned}
& \min \left(C_{p, 1.0 Q_{\mathrm{d}}}^{\mathrm{V} 1}\right) \\
& \text { subject to }\left\{\begin{array}{l}
\eta_{1.0 Q_{\mathrm{d}}}^{O P T}>\eta_{1.0 Q_{\mathrm{d}}}^{O R I} \\
H_{1.0 Q_{\mathrm{d}}}^{O P T} \geq 0.90 H_{1.0 Q_{\mathrm{d}}}^{O R I}
\end{array}\right.
\end{aligned}
$$

Where $C_{p}^{\mathrm{V} 1}$ is the PFIC at the monitor V1 (see Eq. (2) and Fig. 3), $\eta$ is the efficiency and $H$ is the head. The superscript $O P T$ and $O R I$ represent the cases generated during the optimization process and the original case, respectively. The subscript $1.0 Q_{\mathrm{d}}$ indicates the nominal condition.

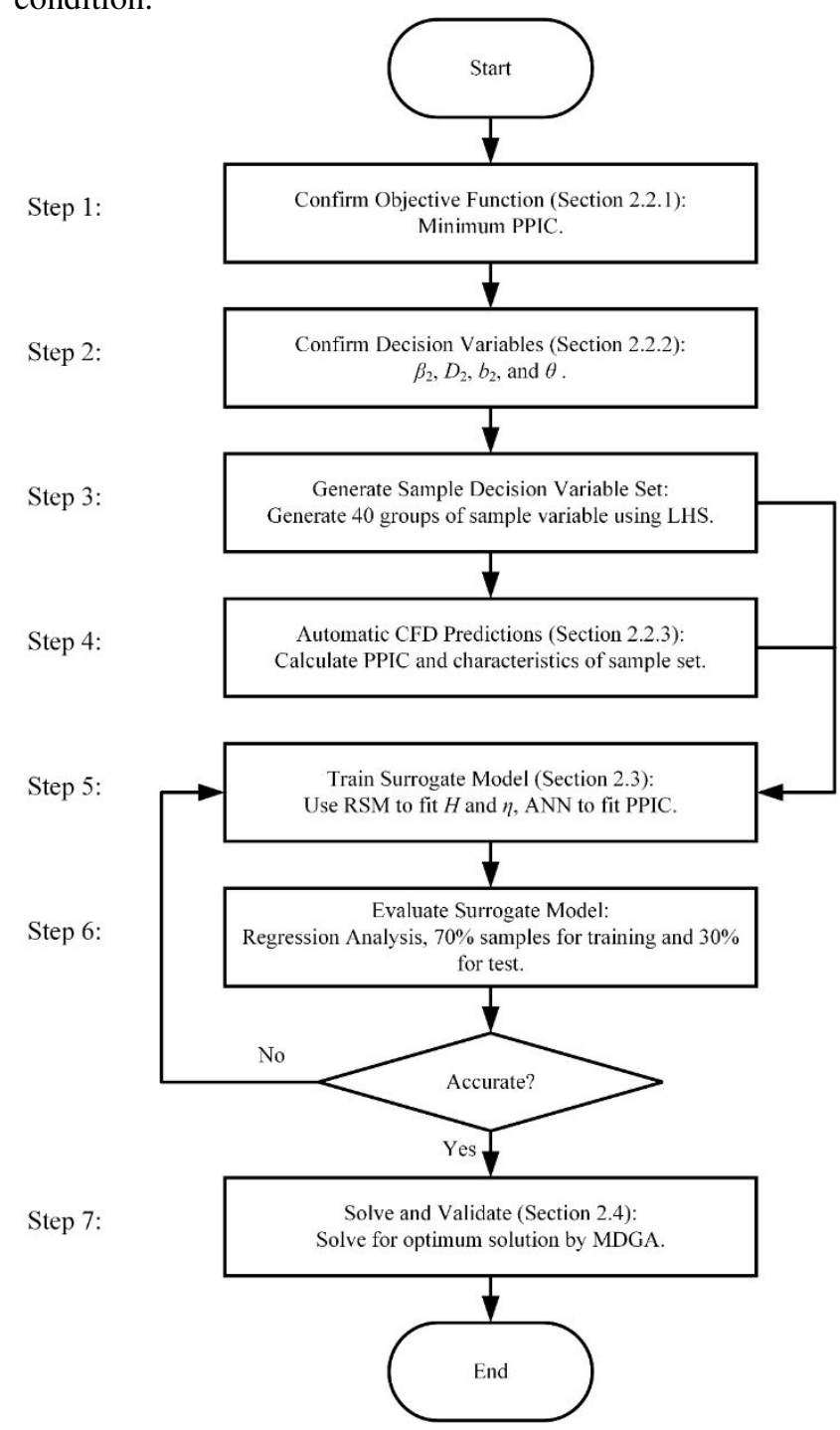

Figure 4 Optimization procedure 


\subsubsection{Decision variables}

The aim of the research is to improve the stability and unsteady performance of the inline pump. It is well known that the impeller is the only working component in the pump, which shows the greatest impact on performance. Therefore, four design variables of the impeller were chosen as the decision variables through the data mining process, whose ranges are listed in Table. 4.

Table 4 Decision variables and boundaries

\begin{tabular}{cccc}
\hline Parameter & Abbreviation & Range & Unit \\
\hline $\begin{array}{c}\text { Blade angle at trailing } \\
\text { edge }\end{array}$ & $\beta_{2}$ & {$[20,40]$} & degree \\
Impeller outlet diameter & $D_{2}$ & {$[128,134]$} & $\mathrm{mm}$ \\
Impeller outlet width & $b 2$ & {$[15,20]$} & $\mathrm{mm}$ \\
Blade wrapping angle & $\theta$ & {$[80,120]$} & degree
\end{tabular}

\subsubsection{Automatic Simulation Technique}

An automatic simulation program for the inline pump was proposed based on MATLAB code and ANSYS WorkBench. The geometries of the impeller cases were generated using ANSYS BladeGen parametrically and then meshed using ANSYS TurboGrid. Finally, the CFD cases were created and calculated by ANSYS CFX[7].

\subsubsection{Design of experiment}

The Latin Hypercube Sampling (LHS) method was used to generate 40 sets of valid design samples selected in the discrete variable decision space. The performance data and pressure fluctuation intensity by unsteady simulation were shown in Table. 5.

Table 5 Sampling data and simulated results

\subsection{Surrogate model}

\subsubsection{Response surface model}

Response Surface Model (RSM) combines experimental design and mathematical modeling, which can fit the mathematical relationship between the target value and the design variables through fewer trials, and construct the equation between the optimization target and the design variables so that the approximate model is closer to the real functional relationship between them[31]. There are also many studies reported that the RSM method performs well in predicting the characteristic of pumps such as efficiency and head [6,9].

In this research, the RSM method was applied to fit the relationship between the external characteristics and the decision variables (refer to section 2.2.2), and the final prediction functions are given in the Eqs. (4) and (5).

$$
\begin{aligned}
\eta= & -326.48+2.22 \beta_{2}+4.65 D_{2}+4.03 b_{2}+0.99 \theta \\
& -0.0086 \beta_{2}{ }^{2}-0.0147 D_{2}{ }^{2}-0.0691 b_{2}^{2}-0.0015 \theta^{2} \\
& -0.0096 \beta_{2} D_{2}-0.0027 \beta_{2} b_{2}-0.0029 \beta_{2} \theta-0.0188 D_{2} b_{2} \\
& -0.0047 D_{2} \theta+0.0075 b_{2} \theta \\
H= & 265.53+1.73 \beta_{2}-4.49 D_{2}-1.08 b_{2}+0.05 \theta \\
& -0.0079 \beta_{2}^{2}+0.0183 D_{2}^{2}-0.0002 b_{2}^{2}-1.1228 \theta^{2} \\
& -0.0060 \beta_{2} D_{2}-0.0096 \beta_{2} b_{2}-0.0017 \beta_{2} \theta+0.1403 D_{2} b_{2} \\
& +0.0002 D_{2} \theta-0.0012 b_{2} \theta
\end{aligned}
$$

In addition, to evaluate the reliability of the model, a regression analysis was carried out. The sample case set was divided into two groups: $70 \%$ of them were used to train the model, and the last $30 \%$ were used for the test. The overall $R^{2}$ (the definition is given as the Eq. (6)) of these two models are 0.9502 and 0.9976, respectively.

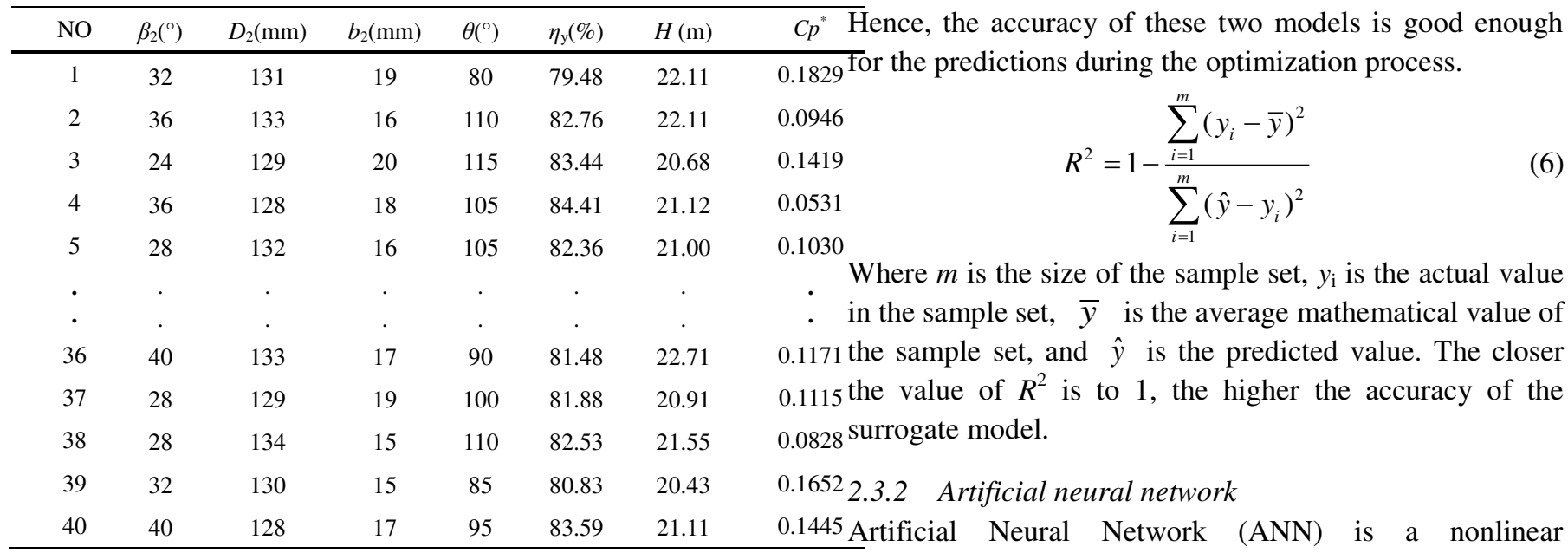
mathematical model of distributed parallel information processing abstracted from neural networks of the brain, 
with high fault tolerance, self-learning, self-adaptive and associative features, widely used in signal processing, artificial intelligence, pattern recognition, automatic control, and other research fields[32].

As mentioned in section 1 , the relationship between inner flow features and decision variables shows a strong nonlinearity, and it is hard to predict accurately. Hence, to fix this situation, a feedforward network with two hidden layers was applied to fit the relationship between PFIC and the decision variables.

A regression analysis was also applied to ensure the stability of the trained model. The result indicated that the $R^{2}$ of the ANN obtained is 0.9303 (Fig. 5), which showed great predicting precision.

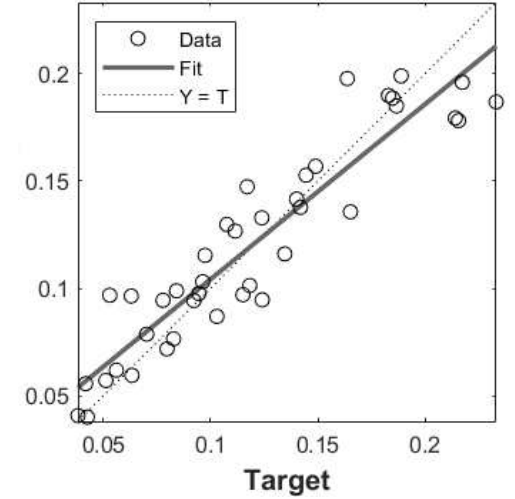

Figure 5 Regression analysis of multi-layer feedforward artificial neural network

\subsection{Modified discrete genetic algorithm}

Genetic algorithm (GA) is biological evolutionary models based on Charles Darwin's theory of natural selection and are widely used in signal processing, machine learning, combinatorial optimization, adaptive control, and artificial life[33]. For the optimization problem of unsteady characteristics of inline pumps with discrete variables, this section proposes a modified discrete genetic algorithm (MDGA) based on the classical genetic algorithm with a binary encoding of discrete fetching positions.

\subsubsection{Modifications}

Based on the classical genetic algorithm, this research proposed two main modifications: (1) position encoding;

(2) adaptive genetic factors.

(1) Position encoding

Define DM as an n-dimensional bounded discrete space, and $M$ represents a two-dimensional optional value array, as shown in the Eq. (7).

$$
M=\left\{\begin{array}{l}
\left\{v a l_{11}, v a l_{12}, \cdots, v l_{1_{1}}\right\} \\
\left\{v_{21}, v a l_{22}, \cdots, v_{2 l_{2}}\right\} \\
\ldots \\
\left\{v a l_{m 1}, v_{m 2}, \cdots, v l_{m l_{m}}\right\}
\end{array}\right\}
$$

Where $m$ is the number of decision variables, $l_{\mathrm{i}}$ is the number of optional values of the $i$-th decision variables, val means optional value.

That is, for the $i$-th decision variables, there are li optional values. In this research, the position index of each optional value was encoding as the binary description for further optimizations. An example of the encoding logic is shown in Fig. 6.

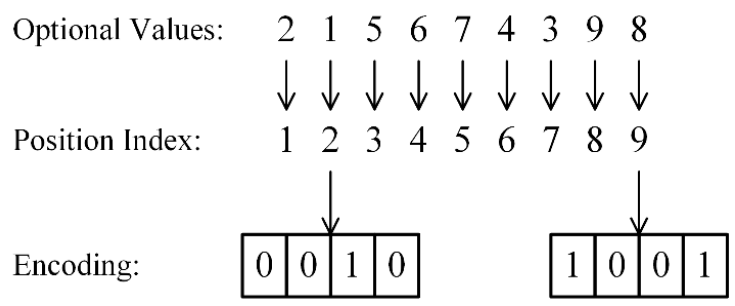

Figure 6 Position encoding

\section{(2) Adaptive genetic factor}

For genetic algorithms, constant crossover and mutation probabilities are executed in the preoperational stage to ensure that enough chromosomes are genetically manipulated to guarantee the diversity of the population, but it also reduces the convergence speed of the algorithm to some extent. After a certain number of iterations, adaptive genetic probabilities are used, the calculation formula is as follows

$$
\begin{gathered}
p_{c}=\left\{\begin{array}{l}
p_{c 0}, f_{c} \geq f_{\text {avg }} \\
p_{c 0} \cdot \cos \left(\frac{f_{\text {min }}-f_{c}}{f_{\text {min }}-f_{\text {avg }}}\right), f_{c}<f_{\text {avg }}
\end{array}\right. \\
p_{m}=\left\{\begin{array}{l}
p_{m 0}, f_{m} \geq f_{\text {avg }} \\
p_{m 0} \cdot \cos \left(\frac{f_{\text {min }}-f_{m}}{f_{\text {min }}-f_{\text {avg }}}\right), f_{m}<f_{\text {avg }}
\end{array}\right. \\
f_{\text {avg }}=\frac{\sum_{i=1}^{N} f_{i}}{N}
\end{gathered}
$$

where $p_{\mathrm{c}}, p_{\mathrm{m}}$ are the adaptive crossover and mutation probabilities, respectively, $p_{\mathrm{c} 0}$ and $p_{\mathrm{m} 0}$ are the initial crossover and mutation probabilities, respectively, $f_{\mathrm{c}}$ is the smaller fitness value of the two chromosomes that have been paired to crossover, $f_{\mathrm{m}}$ is the fitness value of the 
chromosome to be mutated, $f_{\min }$ is the minimum fitness value in the population, and $f_{\text {avg }}$ is the average fitness value of the population, $N$ is the population size, $f_{\mathrm{i}}$ is the fitness value of the $i$-th chromosome.

\subsubsection{Benchmark}

To evaluate the performance of the MDGA, a test on four different benchmark functions was carried out with a comparison to the original genetic algorithm. The specifications of each benchmark function are listed in Table. 6. Two performance indices were utilized: convergence rate $(\mathrm{CR})$ and search speed $(\mathrm{SS})$. In addition, each test was repeated 20 times to ensure the reliability of the results. The maximum iteration was set to 1000 , and the population size $\mathrm{N}$ was set to 100 .

The test results are listed in Table. 7. In terms of convergence rate, for each test function, MDGA achieves $100 \%$, i.e., MDGA finds the global optimum, while only the Drop-Wave function of GA achieves $25 \%$ convergence rate, and the other three test functions do not accurately find the theoretical optimum within the maximum number of iterations. In addition, for most of the cases, MDGA could converge in less than 100 iterations, which shows amazing search speed and is more suitable for the optimization problem in this research.

Table 6 Benchmark function specifications

\begin{tabular}{|c|c|c|c|c|c|}
\hline Name & Formula & Dim & $\begin{array}{c}\text { Decision } \\
\text { Domain }\end{array}$ & Optimum Variable & $\begin{array}{c}\text { Global } \\
\text { Optimum }\end{array}$ \\
\hline Ackley & $f(x)=-a \exp \left(-b \sqrt{\frac{1}{d} \sum_{i=1}^{d} x_{i}^{2}}\right)-\exp \left(\frac{1}{d} \sum_{i=1}^{d} \cos \left(c x_{i}\right)\right)+a+\exp (1)$ & 6 & {$[-32.768,2.768]$} & {$[0, \ldots \ldots, 0]$} & 0 \\
\hline Bulkin N.6 & $f(x)=100 \sqrt{\left|x_{2}-0.01 x_{1}^{2}\right|}+0.01\left|x_{1}+10\right|$ & 2 & $\begin{array}{c}x_{1} \in[-15,-5] \\
x_{2} \in[-3,3]\end{array}$ & {$[-10,1]$} & 0 \\
\hline Drop- Wave & $f(x)=-\frac{1+\cos \left(12 \sqrt{x_{1}^{2}+x_{2}^{2}}\right)}{0.5\left(x_{1}^{2}+x_{2}^{2}\right)+2}$ & 2 & {$[-5.12,5.12]$} & {$[0,0]$} & -1 \\
\hline Griewank & $f(x)=\sum_{i=1}^{d} \frac{x_{i}^{2}}{4000}-\prod_{i=1}^{d} \cos \left(\frac{x_{i}}{\sqrt{i}}\right)+1$ & 6 & {$[-600,600]$} & {$[0, \ldots \ldots, 0]$} & 0 \\
\hline
\end{tabular}

Table 7 Test results for comparison of MDGA and original GA

\begin{tabular}{cccccccc}
\hline \multirow{2}{*}{ Function } & Algorithm & $\mathrm{CR}$ & $\mathrm{SS}_{\text {avg }}$ & $\mathrm{SS}_{\text {min }}$ & $\mathrm{SS}_{\text {max }}$ & $\mathrm{SS}_{\text {median }}$ & $\mathrm{SS}_{\text {std }}$ \\
\hline \multirow{2}{*}{ Ackley } & GA & 0 & - & - & - & & - \\
& MDGA & $100 \%$ & 90.2 & 29 & 311 & 64.5 & 69.86 \\
\hline \multirow{2}{*}{ Bulkin N.6 } & GA & 0 & - & - & - & & - \\
& MDGA & $100 \%$ & 6.9 & 2 & 52 & 3 & 11.37 \\
\hline \multirow{2}{*}{ Drop-Wave } & GA & $25 \%$ & 229 & 130 & 538 & 151 & 174.23 \\
& MDGA & $100 \%$ & 7.45 & 2 & 42 & 4 & 9.65 \\
\hline \multirow{2}{*}{ Griewank } & GA & 0 & - & - & - & & - \\
& MDGA & $100 \%$ & 72.35 & 23 & 129 & 73 & 32.76 \\
\hline
\end{tabular}

Note: the subscript "avg" means the average mathematical value of 20 repeated tests, "min" and "max" represent the minimum and maximum value, "median" means the median value of the results, and "std" means the standard deviation of the results.

\section{Experimental Validation}

\subsubsection{Test rig setup}

To verify the rationality and accuracy of the numerical simulation, the external characteristics test and pressure fluctuation measurement test were conducted on an open test rig in the fluid machinery and engineering laboratory of Jiangsu University. The schematic diagram of the test rig is shown in Fig. 7, and the pressure sensor positions are given in Fig. 8. The test equipment mainly includes import and export pressure gauge, pipeline pump, motor, 
frequency controller, flow meter, throttle valve, etc. The pump inlet pipeline diameter is $80 \mathrm{~mm}$, and the outlet pipeline diameter is $80 \mathrm{~mm}$. The specifications of the experimental sensors are listed in Table. 8.

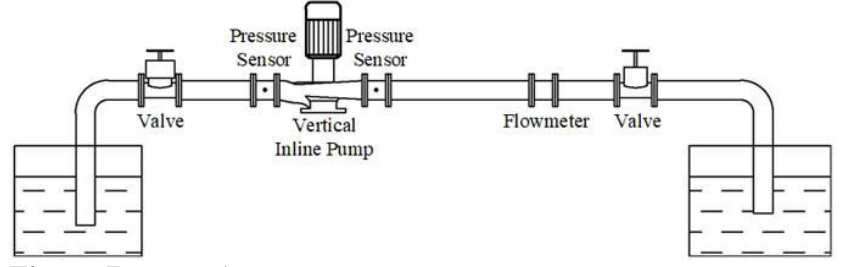

Figure 7 Test rig

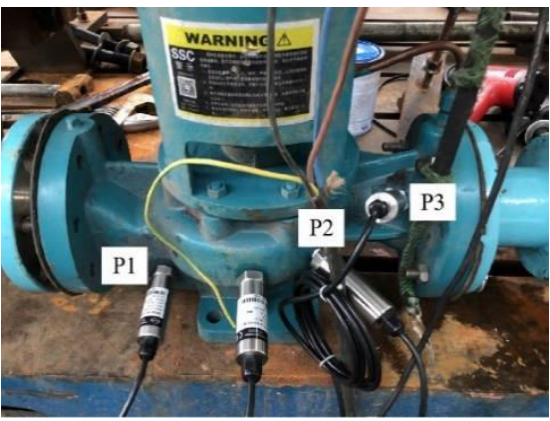

Figure 8 Positions of pressure sensors

Table 8 Experimental sensors specifications

\begin{tabular}{|c|c|c|c|c|}
\hline Parameter & Sensor & Model & Range & Uncertainty \\
\hline Inlet pressure & $\begin{array}{c}\text { Pressure } \\
\text { sensor }\end{array}$ & HM90 & $0-0.2 \mathrm{MPa}$ & $\pm 0.1 \%$ \\
\hline Pressure at P2 & $\begin{array}{c}\text { Pressure } \\
\text { sensor }\end{array}$ & HM90 & $0-0.7 \mathrm{MPa}$ & $\pm 0.1 \%$ \\
\hline Flowrate & $\begin{array}{l}\text { Turbine } \\
\text { flowmeter }\end{array}$ & LW-80 & $12-120 \mathrm{~m}^{3} / \mathrm{h}$ & $\pm 0.5 \%$ \\
\hline $\begin{array}{c}\text { Outlet } \\
\text { pressure }\end{array}$ & $\begin{array}{c}\text { Pressure } \\
\text { sensor }\end{array}$ & WIKA & $0-1 \mathrm{MPa}$ & $\pm 0.1 \%$ \\
\hline
\end{tabular}

\subsubsection{Result comparison}

A comparison between the experimental results and the computational results of the original case was carried out, and the results are given in Fig. 9 and Fig. 10.

As shown in Fig. 9, the computational results and the experimental results showed a good agreement. For the nominal operating condition, the deviation between the computational head and the experimental head is $4.48 \%$, and the deviation of efficiency is $4.81 \%$. Hence, the CFD predictions had a good accuracy, which is acceptable for the further optimization process.

On the other hand, in order to facilitate the analysis of the frequency domain characteristics of the signal, the pressure fluctuation signal obtained from the experimental and numerical simulations is subjected to Fast Fourier Transform (FFT), and the power spectrum density (PSD) is calculated by the Hanning window function. the shaft frequency $f_{\mathrm{n}}=\mathrm{n} / 60=48.5 \mathrm{~Hz}$, and the blade passing frequency $f_{\mathrm{bp}} \mathrm{f}=\mathrm{z} \times f_{\mathrm{n}}=291 \mathrm{~Hz} . f / f_{\mathrm{n}}$ is used to show the rotational frequency multiplier of the blade.

The main frequency of the pressure fluctuation at the monitoring point $\mathrm{P} 2$ under different working conditions is the blade passing frequency (BPF) because the pressure fluctuation near the tongue is mainly due to the rotor-stator interaction. As the flow rate increases, the amplitude of the frequency gradually increased. The change of the amplitude of the blade passing frequency under the small flow rate and the design flow rate was low, but the figure for the overload condition rose by $578.5 \%$ compared to the design condition. It is obvious that the regularity of the numerical results is consistent with the experimental results, but the amplitude prediction was not accurate enough at the nominal condition. However, the performance was good for the part-load and overload conditions.

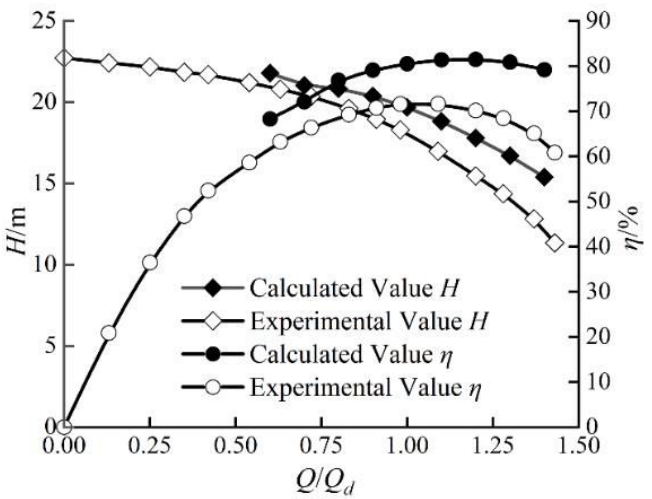

Figure 9 Comparison of performance curves

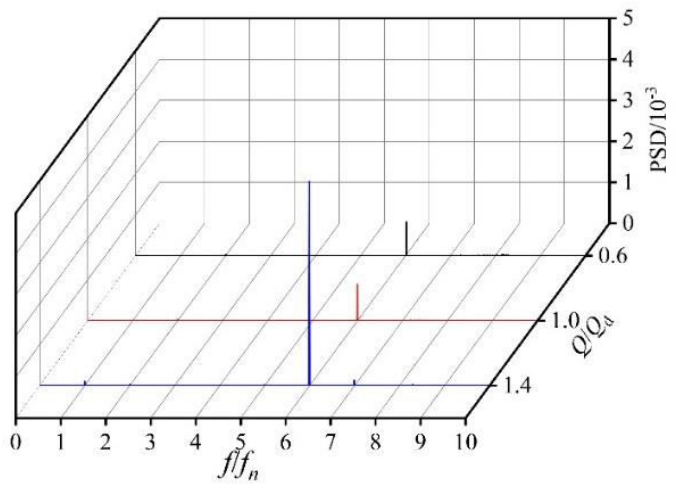

(a) Experimental results 


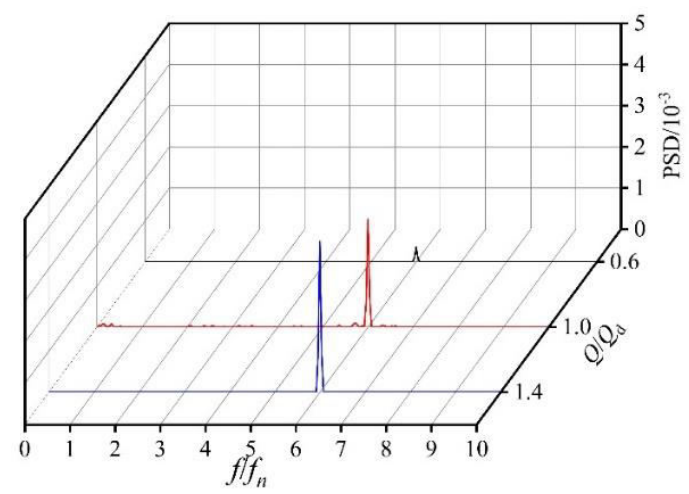

(b) Computational results

Figure 10 Comparison of pressure fluctuation results (P2, the point near the tongue)

\section{Results and Discussions}

\subsection{Performance comparison}

An optimization scheme with better comprehensive performance was selected based on MDGA algorithm searching results and validated by numerical simulation for three operating conditions: $0.6 Q_{\mathrm{d}}, 1.0 Q_{\mathrm{d}}$, and $1.4 Q_{\mathrm{d}}$. The design parameters between the original case and the optimized case are listed in Table. 9. The comparison of the performance is shown in Table. 10.

The results of the optimized case predicted by the surrogate models (refer to section 2.3) are
$\hat{C}_{p, 1.0 Q_{\mathrm{d}}}^{\mathrm{v} 1}=0.0451 \quad, \quad \hat{\eta}_{1.0 Q_{\mathrm{d}}}=81.95 \% \quad$,

$\hat{H}_{1.0 Q_{d}}=19.53 \mathrm{~m}$. It can be found that the deviations between the predicted values and computational values of the efficiency and the head at the nominal condition are $0.35 \%$ and $3.10 \%$, respectively, and the error for PFIC is $15.36 \%$.

After optimization, the efficiency under all investigated conditions is improved significantly, and a maximum efficiency increase of $2.97 \%$ is obtained under the nominal condition. Compared with the original model, the optimized model has a smaller outlet blade angle, impeller outlet diameter and width, and a larger vane wrap angle. For the head, under the selected operating conditions, it has different degrees of reduction, and the largest head drop is found under overload conditions, while it still meets the design requirements. In addition, the PFIC decreases significantly at the nominal and over-load condition, while the difference is small for the part-load condition.

Table 9 Design variables of the original case and the optimized case

\begin{tabular}{ccccc}
\hline Parameter & $\beta_{2}\left({ }^{\circ}\right)$ & $D_{2}(\mathrm{~mm})$ & $b_{2}(\mathrm{~mm})$ & $\theta\left(^{\circ}\right)$ \\
\hline Original case & 23 & 136 & 17.8 & 110 \\
Optimized case & 20 & 133 & 15 & 120 \\
\hline
\end{tabular}

Table 10 Characteristic comparisons between the original case and the optimized case

\begin{tabular}{cccccccccc}
\hline Parameter & $\eta_{0.6 Q_{\mathrm{d}}}$ & $\eta_{1.0 Q_{\mathrm{d}}}$ & $\eta_{1.4 Q_{\mathrm{d}}}$ & $H_{0.6 Q_{\mathrm{d}}}$ & $H_{1.0 Q_{\mathrm{d}}}$ & $H_{1.4 Q_{\mathrm{d}}}$ & $C_{0.6 Q_{\mathrm{d}}}^{\mathrm{V} 1}$ & $C_{1.0 Q_{\mathrm{d}}}^{\mathrm{V} 1}$ & $C_{1.4 Q_{\mathrm{d}}}^{\mathrm{V} 1}$ \\
\hline Original case & 67.34 & 79.86 & 77.84 & 21.51 & 19.42 & 15.00 & 0.1620 & 0.0887 & 0.0896 \\
Optimized case & 69.25 & 82.23 & 79.55 & 21.29 & 18.94 & 14.24 & 0.1649 & 0.0533 & 0.0716 \\
Change & $2.84 \%$ & $2.97 \%$ & $2.20 \%$ & $-1.02 \%$ & $-2.47 \%$ & $-5.07 \%$ & $1.79 \%$ & $-39.91 \%$ & $-20.09 \%$ \\
\hline
\end{tabular}

\subsection{Flow analysis}

\subsubsection{Volute}

In order to understand the flow distribution in the volute, ten cross-sections were defined, and the positions of these sections are shown in Fig. 11. Fig. 12 shows the velocity distribution on the midsection of the volute for the original and optimized cases. The comparison of the PFIC distributions in the original model and the optimized model is illustrated in Fig. 13 and Fig. 14.

For the velocity distribution (see Fig. 12), the flow in the spiral section of the volute is more uniform, and the changes are mainly concentrated in the diffusion tube under the part-load condition. In the original model, the low-velocity area in the diffusion tube is larger, which changes little after optimization, but the flow distribution became more uniform, and the velocity in the mainstream area increased slightly. For the nominal condition, the flow separation can be observed in the diffusion tube of the original model, which disappears after optimization, and the near-wall velocity decreases. In the overload condition, a small area of flow separation is found on the outside of the tongue, which is improved after optimization, and the velocity in the mainstream area increases.

As shown in Fig. 13 and Fig. 14, under the part-load condition, the high PFIC region is mainly concentrated on the area near section II, and the highest PFIC was found near the inner side of the tongue. After optimization, the PFIC at the volute inlet and near the tongue is significantly reduced due to the decrease of the working capacity of the impeller. Meanwhile, the PFIC gradient reduces, and the high PFIC area also becomes smaller after optimization. Similarly, for the nominal condition, the PFIC near the interface and tongue decreases dramatically after optimization, the high PFIC region around the tongue nearly disappears, and more uniform pressure distribution 
is achieved. Under the overload condition, the overall PFIC in the volute is decreased after optimization, and the low PFIC area between section II and section III decreases a lot compared with the original case.
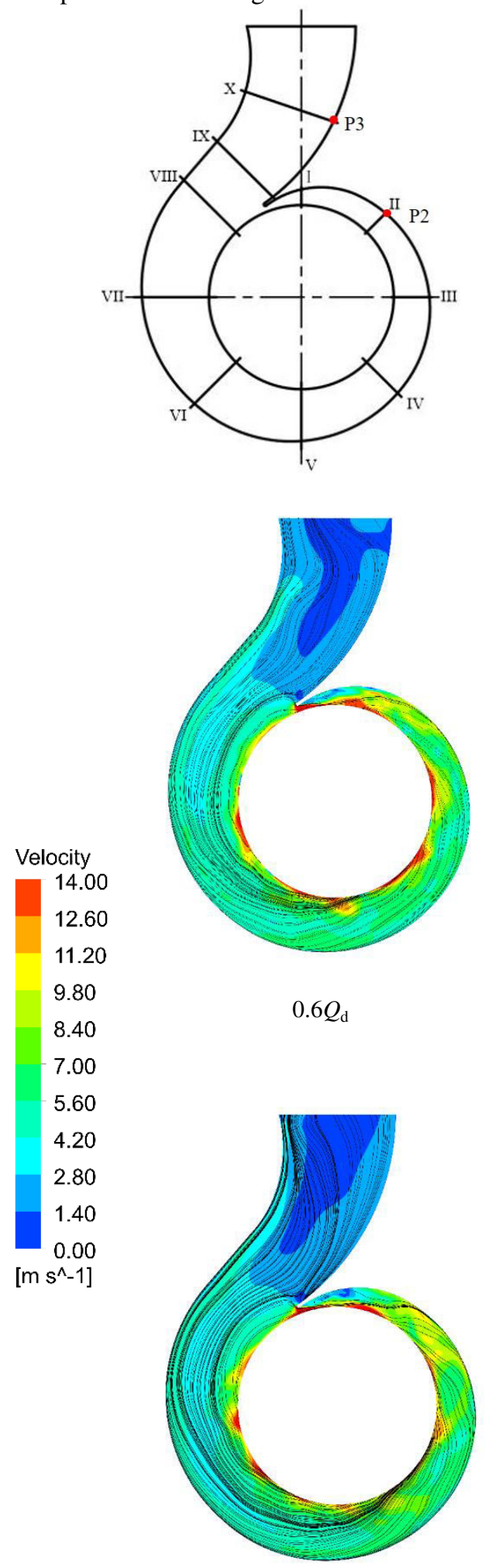

$0.6 Q_{\mathrm{d}}$
Figure 11 Schematic diagram of volute cross-sections

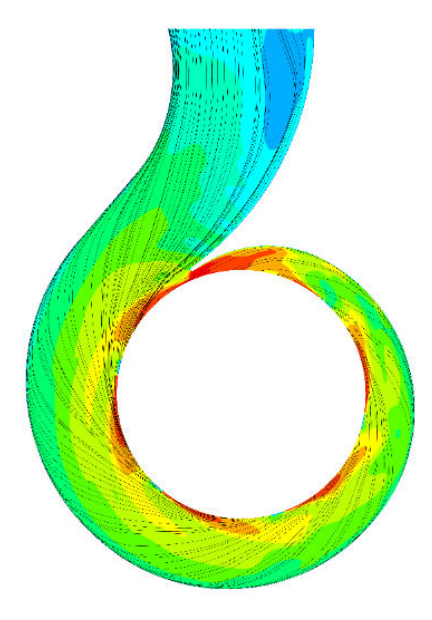

$1.0 Q_{\mathrm{d}}$

(a) Original model

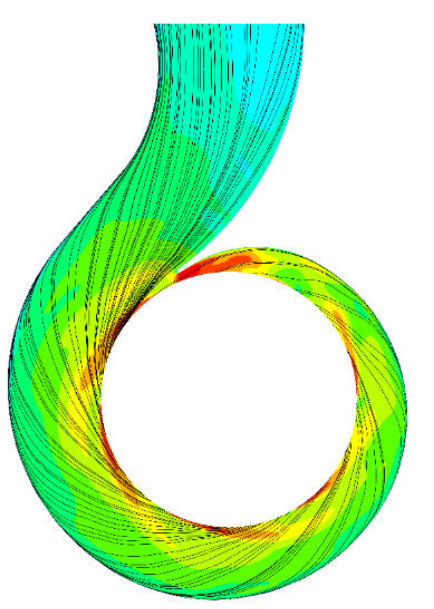

$1.0 Q_{\mathrm{d}}$

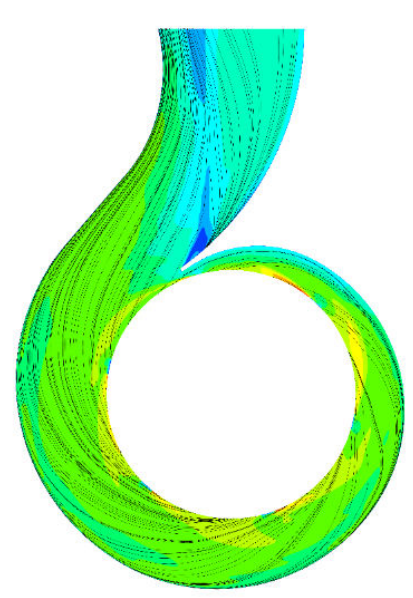

$1.4 Q_{\mathrm{d}}$

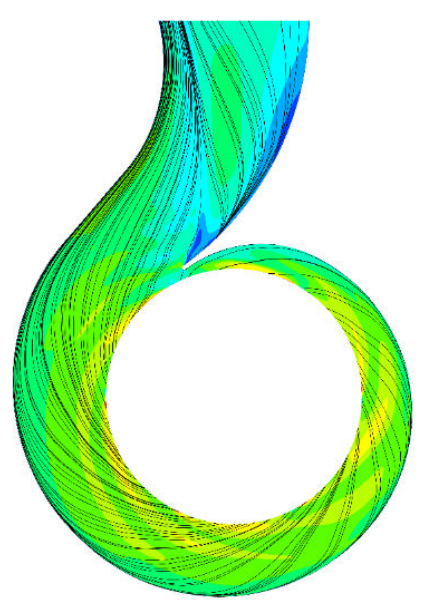

$1.4 Q_{\mathrm{d}}$ 


\section{(b) Optimized model}

Figure 12 Velocity distribution in the volute of the original case and the optimized case

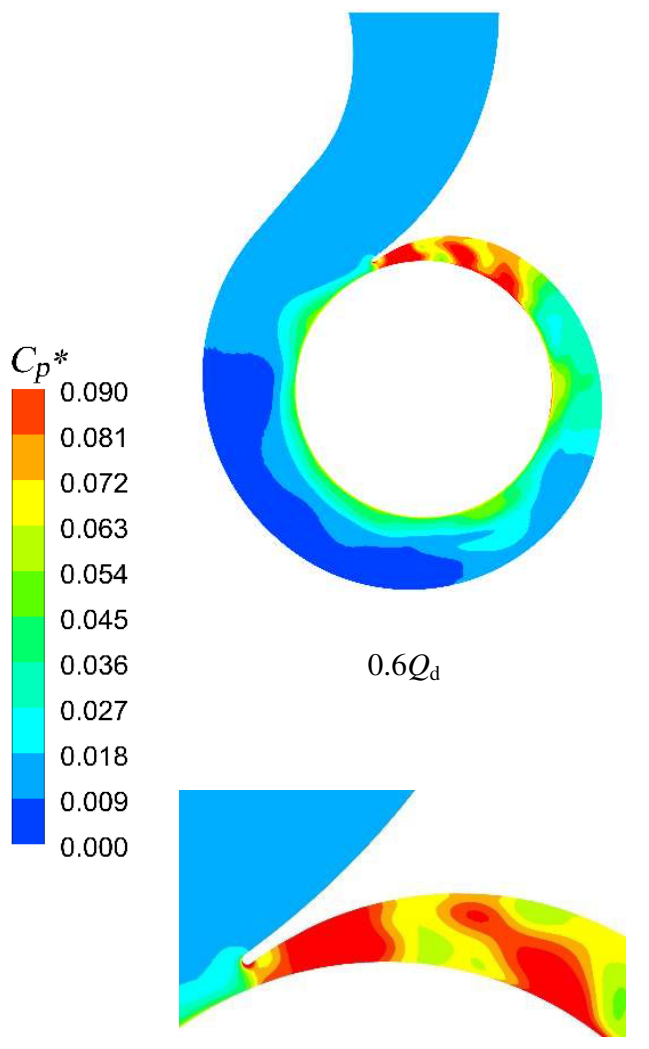

$0.6 Q_{\mathrm{d}}$

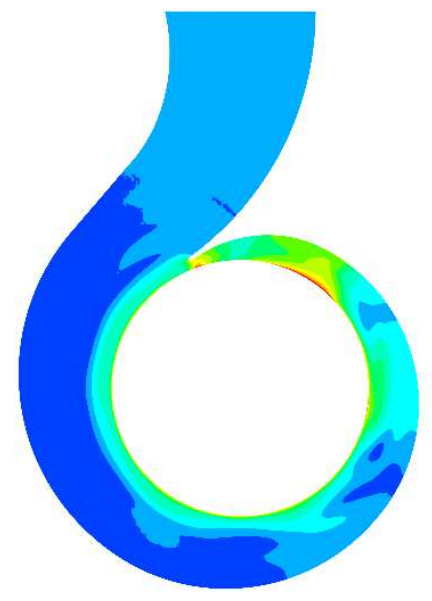

$1.0 Q_{\mathrm{d}}$

(a) Volute

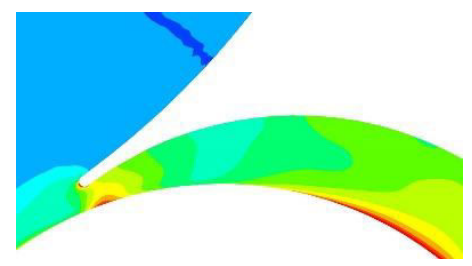

$1.0 Q_{\mathrm{d}}$

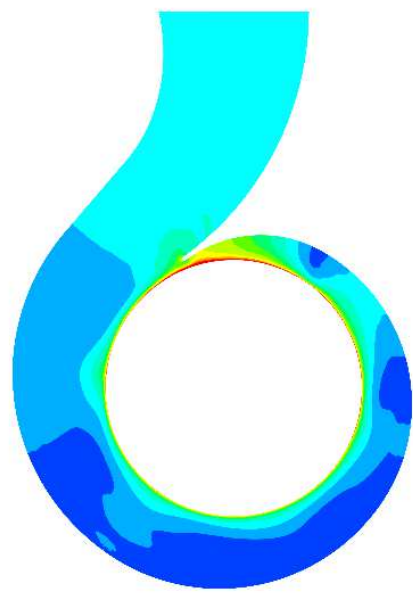

$1.4 Q_{\mathrm{d}}$

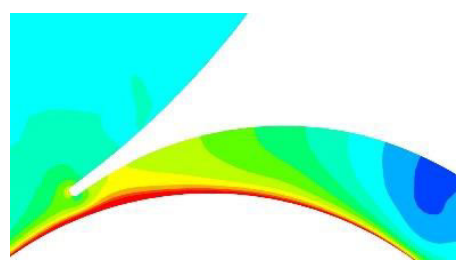

$1.4 Q_{\mathrm{d}}$

(b) Tongue

Figure 13 PFIC distribution in the volute of the original case

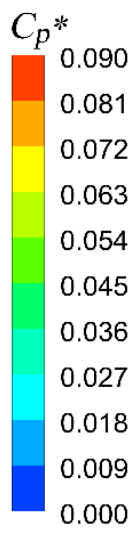

0.000

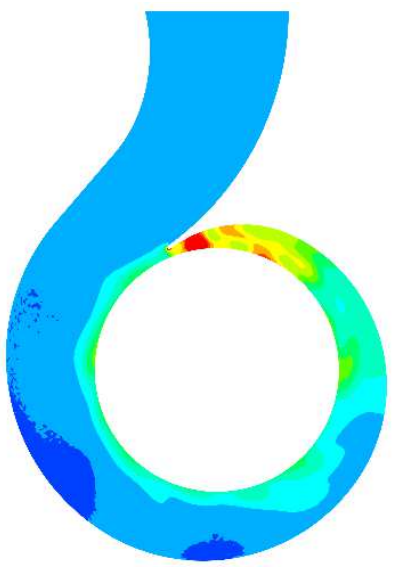

$0.6 Q_{\mathrm{d}}$

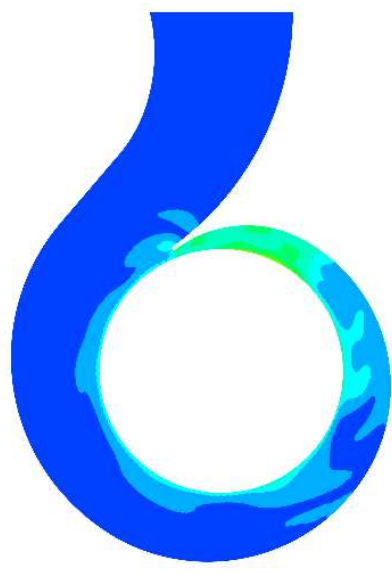

$1.0 Q_{\mathrm{d}}$

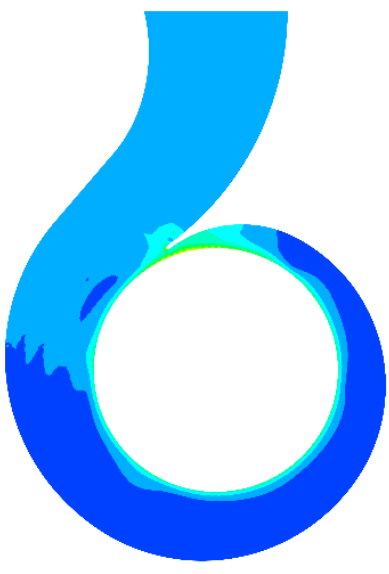

$1.4 Q_{\mathrm{d}}$

(a) Volute 


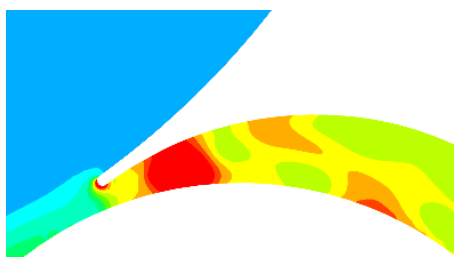

$0.6 Q_{\mathrm{d}}$

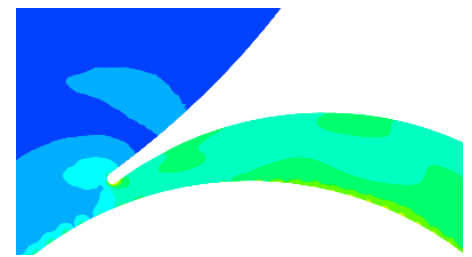

$1.0 Q_{\mathrm{d}}$

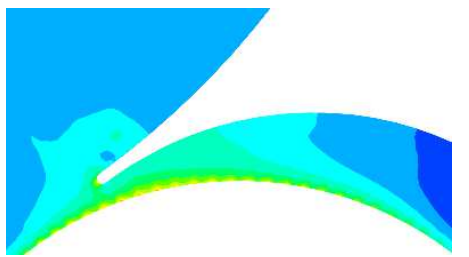

$1.4 Q_{\mathrm{d}}$

(b) Tongue

Figure 14 PFIC distribution in the volute of the optimized case

\subsubsection{Impeller}

The comparison of the velocity distribution on the impeller middle plane between the original case and the optimized case is given in Fig. 15, and the comparison of PFIC distribution is shown in Fig. 16.

It can be seen from Fig. 15 that the flow condition was extremely complicated under the part-load condition. Serious flow separations could be observed on the suction side of the blade. In addition, the recirculation could be found near the impeller outlet, which resulted in the vortices at the suction side and blocked the passage. After optimization, due to the pressure drop in the volute, this phenomenon was improved. For the nominal and overload condition, the velocity gradient in the impeller decreased, and a uniform velocity distribution can be observed after optimization.

From Fig. 16, the PFIC increased from the inlet to the outlet because of the work of the impeller. The low PFIC region was concentrated on the inlet of the impeller. The highest value and the gradient for PFIC were dropped after optimization, especially for the part-load condition, which was mainly due to the improvement of the flow distribution. Also, the low PFIC region in the impeller increased a lot after optimization under both the nominal condition and overload condition.

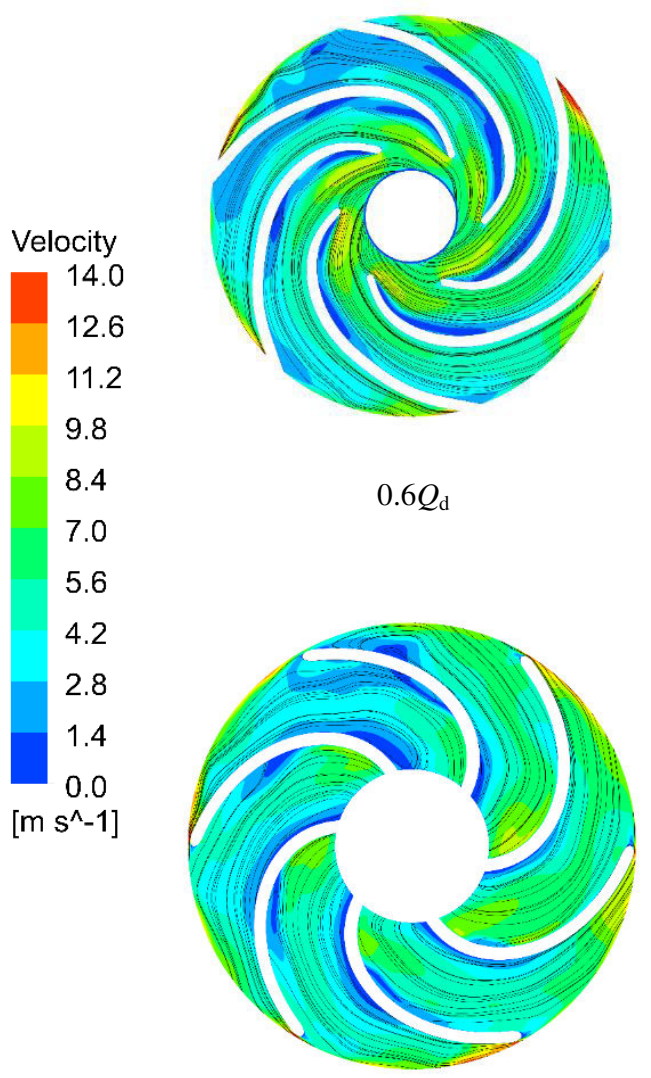

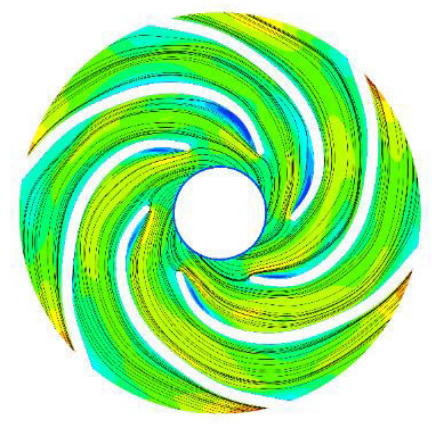

$1.0 Q_{\mathrm{d}}$

(a) Original case

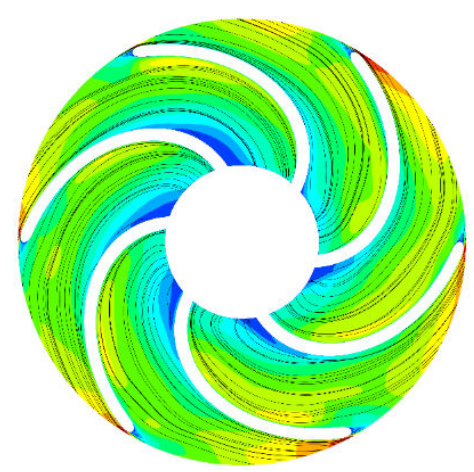

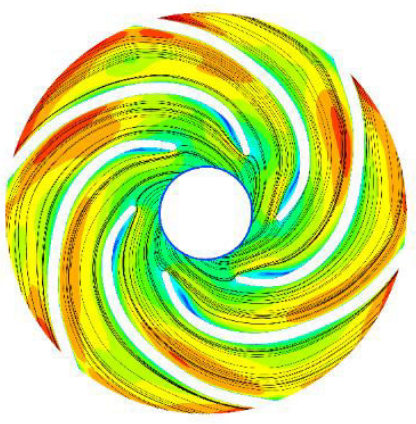

$1.4 Q_{\mathrm{d}}$

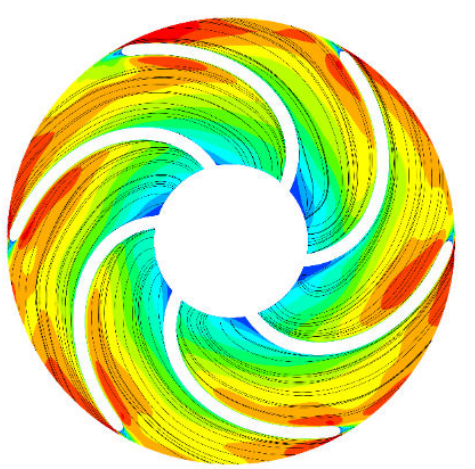


$0.6 Q_{\mathrm{d}}$

$1.0 Q_{\mathrm{d}}$

$1.4 Q_{\mathrm{d}}$

(b) Optimized case

Figure 15 Velocity distributions in the impeller of the original case and the optimized case

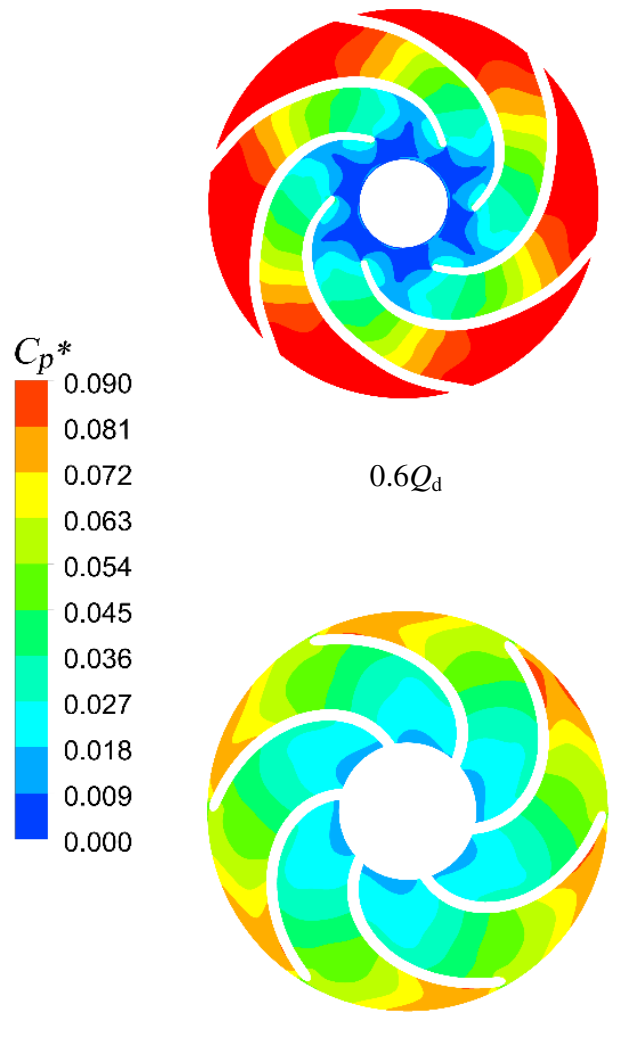

$0.6 Q_{\mathrm{d}}$

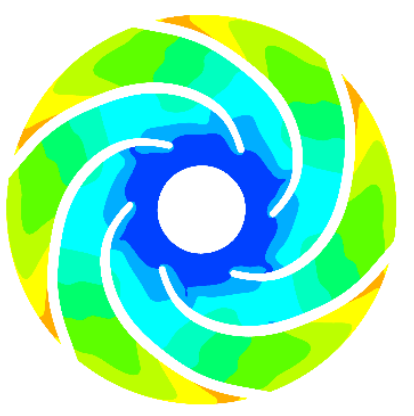

$1.0 Q_{\mathrm{d}}$

(a) Original case

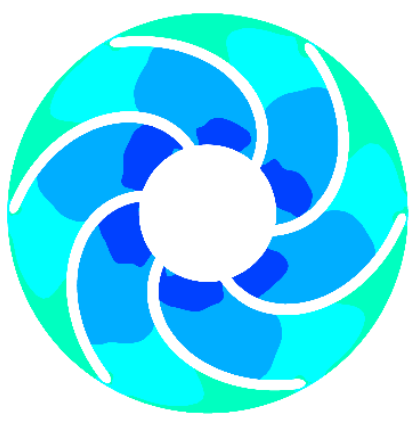

$1.0 Q_{\mathrm{d}}$

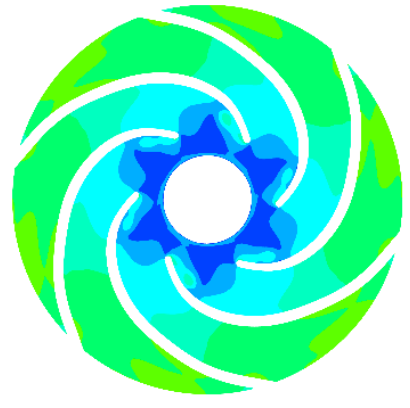

$1.4 Q_{\mathrm{d}}$

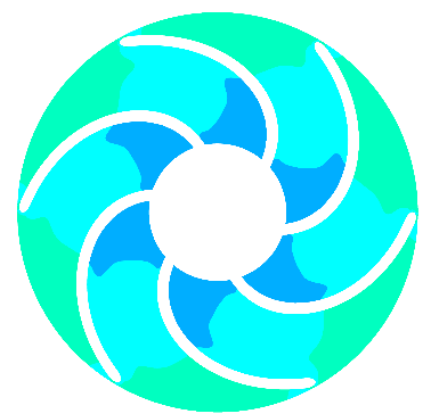

$1.4 Q_{\mathrm{d}}$

(b) Optimized case

Figure 16 PFIC distribution in the impeller of the original case and the optimized case

\section{Conclusions}

In this research, a design optimization of the impeller of the inline pump was carried out to improve the efficiency and working stability based on the transient database. After the data-mining process, four design variables were chosen as the decision variables, and 40 sample cases were generated and calculated by the automatic simulation program in the unsteady state. On the other hand, a modified discrete genetic algorithm was proposed to reduce the optimization cost. Finally, a great improvement in performance was obtained after optimization. The main conclusions of this research are given as follows.

(1) The cost of optimization to improve unsteady characteristics (i.e., PFIC) of pumps is obviously greater than the optimization of steady features (i.e., efficiency), and this problem will be extremely hard if the number of decision variables is large.

(2) The surrogate model performs well in fitting the relationship between the external characteristic parameters and the design parameters. However, its prediction for the internal flow features is still not efficient.

(3) The proposed MDGA has significant advantages over the original GA in terms of search speed.

(4) Although the surrogate model did not perform well in predicting the internal flow properties, it was still a guide for optimization progress. After optimization, 
the performance and stability of the inline pump were significantly improved.

\section{Declaration}

\section{Acknowledgements}

The authors sincerely thanks to 4C-pump for supporting the experimental test and computational source.

\section{Funding}

Supported by Natural Science Foundation of Jiangsu Province (Grant No. BK20190851), Natural Science Foundation of China (Grant No. 51879121), Primary Research \& Development Plan of Jiangsu Province (Grant No. BE2019009-1), Postgraduate Research \& Practice Innovation Program of Jiangsu Province (Grant No. KYCX20_3018), and China Scholarship Council (Grant No. 202008320550).

\section{Availability of data and materials}

The datasets supporting the conclusions of this article are included within the article.

\section{Authors' contributions}

The author' contributions are as follows: Wen-Jie Wang was in charge of the optimization method; Wen-Jie Wang and Qi-Fan Deng wrote the manuscript; Ji Pei was in charge of the review and editing. Jin-Wei Chen focused on the numerical simulation. Qi-Fan Deng and Xing-Cheng Gan worked on the data analysis.

\section{Competing interests}

The authors declare no competing financial interests.

\section{Consent for publication}

Not applicable

\section{Ethics approval and consent to participate}

Not applicable

\section{References}

[1] C Stephen, S Yuan, J Pei, et al. Numerical flow prediction in inlet pipe of vertical inline pump. J Fluids Eng 2018; 140(5): 051201.

[2] S Chu, R Dong, J Katz. Relationship Between Unsteady Flow, Pressure fluctuations, and noise in a centrifugal pump — Part B: effects of blade-tongue interactions. J Fluids Eng 1995; 117(1): 30-35.
[3] Z Wang, Z Qian, J Lu, et al. Effects of flow rate and rotational speed on pressure fluctuations in a double-suction centrifugal pump. Energy 2019; 170: 212-227.

[4] D Fu, F Wang, P Zhou, et al. Impact of impeller stagger angles on pressure fluctuation for a double-Suction centrifugal pump. Chin J Mech Eng 2018; 31(1): 198-211.

[5] D Li, Y,Qin J Wang, et al. Optimization of blade high-pressure edge to reduce pressure fluctuations in pump-turbine hump region. Renewable Energy 2022; 181 : 24-38.

[6] Abdellah El-Hadj A, Abd Rahim S. Optimization of an External Gear Pump using Response Surface Method. J Mech 2020; 36(4): 567-75.

[7] X Gan, J Pei, W Wang, et al. Multi-component optimization of a vertical inline pump based on multi-objective PSO and artificial neural network. J Mech Sci Technol. 2020, 34: 4883-96.

[8] Z Sun, S Xiao, M Xu, et al. Optimization of the structure of water axial piston pump and cavitation of plunger cavity based on the Kriging model. J Vibroengineering. 2016, 18(4): 2460-74.

[9] W Wang, S Yuan, J Pei, et al. Optimization of the diffuser in a centrifugal pump by combining response surface method with multi-island genetic algorithm. Proceedings of the Institution of Mechanical Engineers, Part E: J Proc Mech Eng 2017, 231(2): 191-201.

[10] Z Li, X Zheng. Review of design optimization methods for turbomachinery aerodynamics. Prog Aerosp Sci 2017, 93: $1-23$.

[11] J Madsen, W Shyy, R Haftka. Response surface techniques for diffuser shape optimization. AIAA J 2000, 38: 1512-8.

[12] X Gan, J Pei, S Yuan, et al. Multi-Objective Optimization on Inlet Pipe of a Vertical Inline Pump Based on Genetic Algorithm and Artificial Neural Network. ASME 2018 5th Joint US-European Fluids Engineering Division Summer Meeting. Montreal, Quebec, Canada 2018.

[13] W Wang., M Osman., J Pei, et al. Artificial Neural Networks Approach for a Multi-Objective Cavitation Optimization Design in a Double-Suction Centrifugal Pump. Processes 2019, 7(5), 246.

[14] J Kim, J Choi, K Kim. Design optimization of a centrifugal compressor impeller using radial basis neural network method. ASME Turbo Expo 2009: Power for Land, Sea, and Air: American Society of Mechanical Engineers Digital Collection; 2009. p. 443-51.

[15] Y Zhang, S Hu, J Wu, et al. Modeling and multi-objective optimization of double suction centrifugal pump based on kriging meta-models. Adv Global Optim 2015, 95: 251-61.

[16] J Pei, W Wang, MK Osman, et al. Multiparameter optimization for the nonlinear performance improvement of centrifugal pumps using a multi-layer neural network. J Mech Sci Technol 2019, 33:2681-91.

[17] A Demeulenaere, A Ligout, C Hirsch. Application of multipoint optimization to the design of turbomachinery blades. Turbo Expo: Power for Land, Sea, and Air 2004, 1481-9.

[18] X Gan, W Wang, J Pei, et al. Direct shape optimization and parametric analysis of a vertical inline pump via multi-objective particle swarm optimization. Energies 2020, 13(2): 425. 
[19] R Huang, X Luo, B Ji, et al. Multi-objective optimization of a mixed-flow pump impeller using modified NSGA-II algorithm. Sci China Technol Sci 2015, 58: 2122-30.

[20] S Dhanalakshmi, S Kannan, K Mahadevan, et al. Application of modified NSGA-II algorithm to Combined Economic and Emission Dispatch problem. Int $\mathrm{J}$ Electr Power Energy Syst 2011, 33: 992-1002.

[21] F Miao, H-S Park, C Kim, et al. Swarm intelligence based on modified PSO algorithm for the optimization of axial-flow pump impeller. J Mech Sci Technol 2015, 29: 4867-76.

[22] A Nourbakhsh, H Safikhani, S Derakhshan. The comparison of multi-objective particle swarm optimization and NSGA II algorithm: applications in centrifugal pumps. Eng Optim 2011, 43: 1095-113.

[23] S Derakhshan, M Pourmahdavi, E Abdolahnejad, et al. Numerical shape optimization of a centrifugal pump impeller using artificial bee colony algorithm. Comput Fluids 2013, 81:145-51.

[24] M Namazizadeh, M Talebian Gevari, M Mojaddam, et al. Optimization of the splitter blade configuration and geometry of a centrifugal pump impeller using design of experiment. J Applied Fluid Mech 2020, 13: 89-101.

[25] M Wang, Y Li, J Yuan, et al. Comprehensive improvement of mixed-flow pump impeller based on Multi-Objective Optimization. Processes 2020, 8(8), 905.

[26] J Pei, T Yin, S Yuan, et al. Cavitation optimization for a centrifugal pump impeller by using orthogonal design of experiment. Chin J Mech Eng 2017, 30:103-9.

[27] L Zhou, L Bai, W Li, et al. PIV validation of different turbulence models used for numerical simulation of a centrifugal pump diffuser. Eng Comput 2018, 35:2-17.

[28] ANSYS. ANSYS CFX User's Guide, Release. Canonsburg, PA, USA: ANSYS Inc. 2019.

[29] Y Gu, J Pei, S Yuan, et al. Clocking effect of vaned diffuser on hydraulic performance of high-power pump by using the numerical flow loss visualization method. Energy 2019, 170:986-97.

[30] H Shim, K Kim. Evaluation of rotor-stator interface models for the prediction of the hydraulic and suction performance of a centrifugal pump. J Fluids Eng 2019, 141(11): 111106.

[31] A Khuri, S Mukhopadhyay. Response surface methodology. Wiley Interdisciplinary Reviews: Computational Statistics
2010, 2:128-49.

[32] Z Zhang. Artificial neural network. multivariate time series analysis in climate and environmental research 2018, 1-35.

[33] J Holland. Adaptation in natural and artificial systems: an introductory analysis with applications to biology, control, and artificial intelligence: MIT press; 1992.

\section{Biographical notes}

Wen-Jie Wang, born in 1990, is currently an associate researcher at Jiangsu University, China. He received his $\mathrm{PhD}$ degree from Jiangsu University, China, in 2018. His research interests include intelligent optimization algorithm improvement, hydraulic optimization design of fluid machinery, measurement and analysis of unsteady flow in pumps, and transient flow characteristics of pump turbine.

E-mail: wenjiewang@ujs.edu.cn

Qi-Fan Deng, born in 1994, is currently a $\mathrm{PhD}$ candidate at National Research Center of Pumps, Jiangsu University, China. He received his master degree from Jiangsu University, China, in 2020. His research interests include pump optimization and unstable flow in pump

E-mail: deng_qf@hotmail.com

Ji Pei, born in 1984, is currently a researcher at Jiangsu University, China. He received his $\mathrm{PhD}$ degree from Jiangsu University, China, in 2013. His research interests include fluid machinery (pump) advanced optimization design method, internal flow theory, and multidisciplinary coupling analysis.

Tel: +86-511-88783358; E-mail: jpei@ujs.edu.cn

Jin-Wei Chen, born in 1995, is currently a master candidate at National Research Center of Pumps, Jiangsu University, China.

E-mail: cjwszz@foxmail.com

Xing-Cheng Gan, born in 1994, is currently a PhD candidate at National Research Center of Pumps, Jiangsu University, China. He received his master degree from Jiangsu University, China, in 2019. His research interests include pump system optimization.

E-mail: ganxingcheng@gmail.com 